\title{
Gamification aplicada à formação na indústria hoteleira
}

\author{
Gamification applied to training in the hotel industry
}

\author{
Carla Patrícia Vieira \\ Escola Superior de Hotelaria e Turismo do Estoril, Portugal \\ cpatvieira@gmail.com \\ Maria de Lurdes Calisto \\ Escola Superior de Hotelaria e Turismo do Estoril, Portugal \\ CiTur - Centro de Investigação, Desenvolvimento e Inovação em Turismo \\ lurdes.calisto@eshte.pt \\ Nuno Gustavo \\ Escola Superior de Hotelaria e Turismo do Estoril, Portugal \\ CiTur - Centro de Investigação, Desenvolvimento e Inovação em Turismo \\ nuno.gustavo@eshte.pt
}

\begin{abstract}
Resumo
O presente estudo tem como finalidade a exploração da utilidade de uma plataforma formativa/appmobile assente em gamification para a indústria hoteleira em Portugal, com conteúdos que respondam às necessidades de formação mais comuns neste contexto. Através da gamification, as empresas podem motivar os trabalhadores a participar em processos de aprendizagem e, assim, melhorar a produtividade. Com recurso a uma metodologia de natureza qualitativa, foram auscultados cinco diferentes stakeholders, do lado da oferta formativa e três do lado da procura. Neste caso foram recolhidos dados em três cadeias hoteleiras que operam em Portugal, abrangendo um total de vinte e três trabalhadores, em cinco focus group. Os resultados do estudo permitiram identificar as principais necessidades formativas existentes nas diferentes unidades, os principais obstáculos sentidos pelos gestores e trabalhadores na execução do plano formativo e identificar a gamification aplicada à formação como uma estratégia motivacional e de fomento da adesão à formação.
\end{abstract}

Palavras-chave: Gamification; hotelaria; e-learning; formação.

\begin{abstract}
This study aims to explore the usefulness of a training platform/mobile app based on gamification for the hotel industry in Portugal, with contents that respond to the most common training needs in this context. Through gamification, companies can motivate workers to participate in learning activities and thus improve productivity. Using a qualitative approach, five different stakeholders were surveyed, companies that offer these type of training solutions and three hotel companies. In this case, data were collected in three hotel chains operating in Portugal, from twenty-three employees in five focus groups. The results of the study allowed the identification of the main training needs in the different hotel units and the main obstacles felt by managers and workers in the implementation of the training plan and also identified gamification in training as a motivational strategy that promotes adherence to training.
\end{abstract}

Keywords: Gamification; hospitality; e-learning; training.

Dos Algarves: A Multidisciplinary e-Journal, 34- 2019.

ISBN 2182-5580 @ ESGHT - University of the Algarve, Portugal.

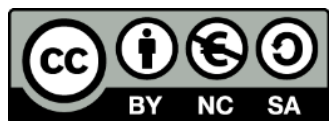

To cite this article: Vieira, C. P., Calisto, M. L. \& Gustavo, N. (2019). Gamification aplicada à formação na indústria hoteleira. Dos Algarves: A Multidisciplinary e-Journal, 34, 81-100. doi: 10.18089/DAMeJ.2019.34.5 


\section{Introdução}

Num momento em que grande parte do que fazemos é mediado pelas tecnologias, jogos digitais e redes sociais, as empresas podem utilizar de forma integrada estas ferramentas para mudar comportamentos, transformando processos tradicionais em experiências inovadoras para muitos dos seus clientes e empregados. Este processo é comumente referido como gamification, que pode ser definida como o uso de elementos de design de jogos para motivar o comportamento de utilizadores, em contextos não-jogo, nomeadamente em contextos organizacionais, aumentando a experiência e o envolvimento do utilizador (Domínguez, Saenz-de-Navarrete, de-Marcos, Fernández-Sanz, Pagés \& Martínez-Herrálzf, 2013; Robson, Plangger, Kietzmann, McCarthy \& Pitt, 2016). Nos últimos anos, a gamification tem sido aplicada em diferentes domínios na tentativa de melhorar os resultados do desenvolvimento das tarefas diárias e do trabalho (Urh, Vukovic, Jereb \& Pintar, 2015) e há um interesse crescente em gamification (Robson et al., 2016). A gamification é um processo que integra as propriedades motivacionais dos jogos nas atividades de aprendizagem, agregando o desejo humano de comunicar e partilhar realizações com o estabelecimento de metas, para direcionar a atenção dos formandos e motivá-los para a ação (Landers, 2014).

É hoje reconhecido que as pessoas representam o capital mais valioso de muitas organizações (Popescu \& Avram, 2012). Para tal, os gestores da área dos recursos humanos $(\mathrm{RH})$ devem focar-se sobre aspetos que atraiam bons profissionais, oferecendo um bom ambiente de trabalho, benefícios atraentes e progressão de carreira. Também é fundamental a utilização de ferramentas motivacionais de forma a obter altos níveis de desempenho e desenvolver a equipa, a fim de melhorar as competências exigidas, através da avaliação de desempenho e da formação contínua do trabalhador. Outro dos aspetos essenciais é manter os trabalhadores mais talentosos através de políticas de retenção e de medidas que evitem a saída destes para empresas concorrentes.

A hotelaria é muitas vezes caracterizada como um setor 'à parte' no que se refere às práticas de gestão de recursos humanos $(\mathrm{GRH})$, onde estas são muitas vezes caracterizadas como inadequadas (Keep \& Mayhew, 1999). Contudo, as poucas empresas com boas práticas, reconhecem que estas melhoram a produtividade e a rentabilidade (Enz \& Siguaw, 2000). Reconhecem também que a formação e o desenvolvimento das pessoas são atividades fundamentais para reduzir lacunas no desempenho e, que equipam os trabalhadores com os conhecimentos, capacidades e atitudes necessárias à execução das suas funções em conformidade com os padrões organizacionais e os requisitos do cliente. A exigência de um serviço de alta qualidade em muitos destinos turísticos, juntamente com a intensidade do trabalho que caracteriza a indústria, resulta na necessidade do setor do turismo recrutar e reter profissionais qualificados, em termos de educação e formação. Esta necessidade tornase mais premente à medida que a geração millennial entra no mercado de trabalho. Os millennials têm afinidade com as tecnologias de informação e comunicação e com a comunicação mediada por computador; veem o trabalho em termos flexíveis (especialmente onde e quando o trabalho está pronto); e desejam horários de trabalho que acomodem os seus desejos de equilíbrio entre trabalho e vida pessoal. As características da geração millenial sugerem a importância da gamification para a indústria hoteleira onde grande parte do mercado de trabalho é, atualmente, desta geração (Randstad Work Solutions, 2007; Simmons, 2008). 
Em Portugal, o número de estabelecimentos hoteleiros cresceu cerca de 120\% entre 2004 e 2016, passando de uma rede de 563 estabelecimentos para 1238. Especificamente, as unidades de 5 estrelas cresceram 94\% em número, de uma evolução de 56 para 109. As unidades de 4 estrelas sofreram um crescimento de 71\%. As unidades de 3 estrelas evidenciaram um crescimento de $26 \%$ e por fim, as unidades de 2 estrelas e 1 estrela evidenciam um crescimento entre 2004 e 2016 de 211\%, quando, em 2004, detinham 111 hotéis e, em 2016, 346 (Turismo de Portugal, 2017).

Relativamente ao pessoal ao serviço no setor, entre 2004 e 2015, este aumentou de 26815 para 34869 pessoas, que representa um acréscimo de 34\% ao longo desses 11 anos. Numa análise mais pormenorizada, é visível um crescimento de $40 \%$ do pessoal ao serviço nas estruturas hoteleiras de 5 estrelas, entre 2009 e 2014. Nas estruturas de 4 estrelas, no período de 2009 a 2015, verificou-se um crescimento de $25 \%$. Inversamente, as estruturas de 3 estrelas diminuíram o número de pessoas em 4\%, passando de 6543 em 2009, para 6237 em 2015. Também os hotéis de 2 estrelas e 1 estrela diminuíram em $24 \%$ o número de trabalhadores, entre 2009-2015, passando de 3183 para 2415 (Turismo de Portugal, 2017).

É possível concluir que esta é uma indústria com dimensão relevante, onde é visível o crescimento em número de estabelecimentos em todas as categorias, e do pessoal ao serviço nas unidades das categorias superiores. É fundamental que o crescimento das unidades hoteleiras seja acompanhado pelo investimento na capacitação das pessoas para assegurar um serviço de qualidade e, simultaneamente, um ambiente saudável e capaz de gerar compromisso e motivação nas equipas.

Apesar das vantagens que muitos identificam na gamification, o setor da hotelaria não a está a aproveitar ao máximo, como outros setores estão a fazer (Negruşa, Toader, Sofică, Tutunea \& Rus, 2015). Assim, este estudo tem como objetivo geral explorar a utilidade de uma plataforma formativa/app-mobile assente em gamification para a indústria hoteleira em Portugal, com conteúdos que respondam às necessidades de formação mais comuns neste contexto.

O estudo realizado é relevante quer do ponto de vista teórico, quer do ponto de vista prático. Em termos académicos, demonstra-se a aplicabilidade do conceito de gamification ao sector hoteleiro em Portugal, através de uma abordagem metodológica que pode ser replicada para outros contextos.

A investigação estruturou-se em dois sub-estudos: 1) do lado da oferta, foram identificadas e auscultadas as empresas que desenvolvem projetos gamification em Portugal, e foram realizadas entrevistas exploratórias; 2) do lado da procura, e de forma a conhecer a recetividade a esta ferramenta e também as necessidades formativas específicas das empresas hoteleiras, foram realizados focus group com gestores das empresas e com trabalhadores do setor hoteleiro.

Do ponto de vista prático, o estudo fornece aos gestores de $\mathrm{RH}$ as vantagens da gamification como uma ferramenta para responder aos desafios que se colocam à hotelaria em Portugal, nomeadamente em termos de gestão da formação. Com base neste trabalho, poderá futuramente ser possível a construção e/ou adaptação de uma plataforma formativa/app-mobile com conteúdos específicos para a área hoteleira com recurso a estratégias de gamification.

Esta plataforma foi pensada para ser uma app mobile e integrar diferentes componentes: 1) desafios; 2) conteúdos; 3) tarefas; 4) reconhecimento e; 5) perfil. A app foi pensada para que 
- utilizador possa avançar no seu percurso (vários níveis), trabalhando diversos conhecimentos e recebendo feedback sobre os mesmos. O utilizador teria à sua disposição um conjunto de conteúdos em formato desafio (quizzes, sopa de letras, caça palavras, puzzles, etc.). As recompensas seriam imediatas e o feedback detalhado e personalizado em função das respostas do formando. Ao avançar nos desafios da app, o utilizador acumularia pontos que podiam (deviam) vir a ser premiados pelo hotel com ofertas especiais. Estes resultados seriam comparados com os de outros participantes.

Existiria também a possibilidade de análise estatística dos resultados e que possuía várias funcionalidades, como por exemplo, gerir utilizadores, desafios, objetivos, iniciativas e parâmetros, entre outros. Também permitiria notificar os resultados alcançados em todas as iniciativas e o nível de utilização da plataforma e por último, conhecer os seus trabalhadores, o seu nível de participação, resultados e conquistas. A aplicação também permitiria a estruturação dos conteúdos, que incluíam desafios (questionários, sopas de letras, caça palavras, etc.) e que uma vez realizados com sucesso contribuem para a aquisição de estrelas. O conceito evolutivo das etapas formativas correspondia à conquista de estrelas (fazendo alusão às estrelas de um hotel que sugerem a qualidade do serviço).

Esta ferramenta poderá vir a apoiar as unidades hoteleiras na formação dos trabalhadores, quer na área técnica quer na comportamental, promovendo a aquisição e desenvolvimento de competências de uma forma lúdica e interativa, consequentemente aumentando a motivação para a formação e os níveis de satisfação dos trabalhadores em relação à formação, com impacto na sua eficácia.

\section{Revisão da literatura}

\subsection{A gamification aplicada à formação}

Existe um crescente interesse dos investigadores sobre o efeito dos jogos digitais na aprendizagem (Erhel \& Jamet, 2013), em parte porque a aprendizagem é uma das áreas onde a gamification está a crescer e ganhar popularidade (Domínguez et al., 2013). A associação da gamification a ambientes digitais de aprendizagem (e-learning), consiste, assim, numa atividade competitiva onde são definidas metas educacionais destinadas a promover a aquisição de conhecimento.

Robson et al. (2016) defendem que há um interesse crescente em gamification - definida como o uso de elementos de design de jogos para motivar o comportamento de utilizadores em contextos não-jogo, nomeadamente em contextos organizacionais. Também para Domínguez et al. (2013), a gamification representa a incorporação de elementos de jogo num software não-jogo para aumentar a experiência e o envolvimento do utilizador.

Para Robson et al. (2016), a literatura académica de negócios oferece pouca direção, ou compreensão da gamification, dos seus princípios de design, e das principais motivações psicológicas subjacentes pelos quais a gamification gera mudanças de comportamento e alcança as metas organizacionais. No entanto, três novos desenvolvimentos permitiram um enriquecimento e inovação a este respeito: (1) novos conhecimentos sobre a conceção e gestão de experiências de jogo; (2) interligação com o nascimento das redes sociais e o desenvolvimento dos telemóveis, portáteis, tablets; e (3) o maior interesse em fornecer mais experiências envolventes (Robson et al., 2016). Em primeiro lugar, ao longo dos últimos vinte anos, com o crescimento e a importância da indústria dos jogos de computador, os game 
designers e investigadores têm investido significativamente em estudos para entender melhor o que faz um jogo de computador envolvente e bem-sucedido. Em segundo lugar, a penetração das redes sociais e das tecnologias móveis e baseadas na web mudou a forma como os indivíduos e as organizações participam, compartilham, cocriam, discutem e modificam qualquer tipo de experiência (Kietzmann, Hermkens, McCarthy \& Silvestre, 2011). Em terceiro, as empresas estão continuamente a procurar novas maneiras de influenciar os comportamentos dos trabalhadores e clientes.

Desde que devidamente concebidas, as atividades de e-learning que utilizam a gamification podem aumentar a satisfação, o engagement, e a eficiência dos formandos. A combinação certa de e-learning, gamification, tarefas equilibradas e competências pode levar os formandos ao chamado estado de fluxo. Csikszentmihalyi (2008) descreve o estado de fluxo como uma experiência ótima caracterizada como um estado de estar totalmente focado e envolvido numa atividade. De acordo com McGonigal (2011), o sentimento de fluxo é desencadeado por quatro elementos que os bons jogos têm em comum: metas, regras, feedback e participação voluntária. Se a dificuldade das tarefas for corretamente equilibrada, pode conduzir os jogadores a um estado de fluxo que é altamente motivador (Csikszentmihalyi, 2008). De acordo com Jackson e Eklund (2002), o fluxo é uma parte importante de atividades onde a concentração de uma pessoa e as suas competências são importantes para um resultado. Em experiências gamified otimizadas, as respostas emocionais dos jogadores e as dinâmicas que emergem durante o jogo moldam os mecanismos que comandam o jogo e vice-versa.

Os três princípios da gamification são a mecânica, dinâmica e emoções (MDE) (Robson et al., 2016).

Existem três tipos diferentes de mecânica - a mecânica de instalação, a mecânica de regras e a mecânica de progressão - que são importantes não só para jogos, mas também para experiências gamified.

A mecânica de instalação são as considerações que moldam o ambiente da experiência, incluindo a definição do conceito, dos objetos necessários e de como os objetos devem ser distribuídos entre os jogadores (Elverdam \& Aarseth, 2007). Os designers devem considerar a dimensão espacial para determinar onde, no mundo real, ou no mundo virtual, a experiência terá lugar. Definem também as dimensões temporais para regular quando a experiência gamified vai acontecer, a sua duração e em que horário acontece. As escolhas de design também incidem sobre o tipo de jogador que pode jogar, se a experiência é para jogadores únicos ou múltiplos, se há uma única equipa ou múltiplas, e se incluem verdadeiros amigos, estranhos, ou aliados, e até inimigos, mesmo que controlados por computador.

A mecânica de regras permite moldar o conceito ou objetivo da experiência gamified a ser perseguido (Elverdam \& Aarseth, 2007). Prescreve as ações que são permitidas, mas também as restrições (por exemplo de tempo) que limitam essas ações, a fim de criar pressão para os jogadores (Kelly, 2012).

A mecânica de progressão pretende descrever diferentes tipos de instrumentos que os designers podem incorporar e a afetar à experiência (Elverdam \& Aarseth, 2007). Para sinalizar o progresso do jogador, são muitas vezes utilizadas as recompensas de realização. Estas podem ser sistemas de pontos de vitória virtuais que os jogadores acumulam à medida que progridem - tais como pontuação, níveis, barras de progresso ou recursos (por exemplo, resistência) - mas podem também ser recompensas reais (por exemplo, financeiras ou ainda 
recompensas com significado social - por exemplo emblemas, troféus, tabelas de classificação).

É importante ressaltar que a mecânica da gamification é fundamental para garantir que os jogadores recebem recompensas que irão motivar a mudança de comportamento desejado e contribuem para a dinâmica e as emoções dos jogadores. Além disso, a compreensão dos desejos e motivações dos jogadores é a chave para projetar experiências gamified envolventes. Para alguns jogadores, a tabela de classificação será eficaz para motivar mudanças de comportamento. Para outros, as oportunidades para colaborar e criar empatia com os outros jogadores é mais atrativo (Robson et al., 2016).

Outro dos princípios MDE, a dinâmica da gamification, corresponde aos comportamentos do jogador que emergem quando as mecânicas são executadas durante a experiência gamified (Robson, Plangger, Kietzmann, McCarthy \& Pitt, 2015). Ao contrário da mecânica que é definida pelos designers, a dinâmica gamification é produzida pela forma como os jogadores seguem a mecânica escolhida pelos designers. Essas dinâmicas descrevem os comportamentos no jogo e as ações estratégicas dos jogadores. Por exemplo, os jogadores são mais competitivos quando sabem que estão a ser observados, pois não querem ser derrotados publicamente.

O terceiro princípio MDE, as emoções, são um produto de como os jogadores seguem a mecânica e geram dinâmicas. As emoções na gamification (por exemplo, excitação e frustração) são os estados afetivos evocados durante a experiência (Robson et al., 2015).

Segundo Robson et al. (2016), a experiência gamified terá de ser monitorizada, e os gestores desempenham um papel fundamental nesta monitorização. Assim, o papel do gestor estende-se para além da conceção da experiência gamified, os gestores devem monitorizar os jogadores para garantir que estes ainda estão envolvidos e não estão a quebrar as regras. Métricas sofisticadas podem não assegurar o cumprimento das regras, daí a importância de o gestor monitorizar a experiência gamified. Por outro lado, sem métricas apropriadas, uma organização não pode medir o progresso ou melhorar experiências passadas.

O MDE ajuda também a esclarecer como os designers e os jogadores percebem experiências gamified de maneira diferente (LeBlanc, 2004). Para os designers de gamification, o foco principal é na seleção da mecânica adequada, a fim de manter o controlo sobre a experiência, seguido por um foco sobre a dinâmica e, finalmente, nos jogadores e suas emoções. Para os jogadores, por outro lado, são essenciais as emoções. A adrenalina, resultante de sobreviver a uma aventura ou dominar um desafio mental, e as dinâmicas associadas, são mais importantes do que as regras que os tornam possível (Lazzaro, 2004).

Em síntese, a compreensão da mecânica da gamification, da dinâmica e das emoções, e como esses princípios se relacionam entre si, são a chave para gamifying uma experiência com sucesso (Lazzaro, 2004).

Os factores intrínsecos são o mais poderoso motivador em gamification, pois leva a um profundo engagement com as atividades e com as outras pessoas. As recompensas intrínsecas batem as recompensas extrínsecas (Xu, Weber \& Buhalis, 2014). Segundo os mesmos autores, as recompensas intrínsecas enquadram-se nas seguintes categorias principais: 1) Relacionamento - necessidade de interagir e de se conectar com outros jogadores dentro do mesmo grupo de interesses, para compartilhar realizações e ser reconhecido entre os pares. McGonigal (2011) definiu esta categoria como 'conexão social', com grande impacto sobre a 
felicidade, e onde os contactos sociais são uma fonte de compartilha de experiências, construir laços e criar memórias. 2) Competência - dá ao jogador a sensação de ter capacidade para dominar o sistema e atingir metas. Os objetivos devem ser claros, visuais, variados e bem estruturados para fornecer motivação e otimismo relativamente às próprias oportunidades e possibilidades. As falhas são necessárias para melhorar a experiência de jogo, e o jogador precisa de ter um feedback positivo para se sentir encorajado. 3) Autonomia - a liberdade do jogador para aderir voluntariamente a um sistema gamified e abandonar a qualquer momento. Se o jogador perde autonomia e é controlado pelo sistema gamified irá conduzir a uma experiência de motivação não inspiradora (Deterding, 2011).

Um estudo relatou uma relação positiva entre o nível de motivação e de aprendizagem intrínsecas num jogo de aprendizagem digital (Liu, Horton, Olmanson \& Toprac, 2011). Outros estudos focaram-se na relevância da teoria do fluxo (Hays, 2005). A motivação intrínseca refere-se ao desejo interior de se envolver numa tarefa por interesse ou diversão, ou até mesmo por causa do desafio que oferece (Erhel \& Jamet, 2013). Para Csikszentmihalyi (1988), o fluxo é a experiência subjetiva imediata que ocorre quando um indivíduo se envolve numa atividade.

Gamification e engagement são tópicos relevantes dentro da literatura de negócios (Kim \& Mauborgne, 2014; Robson et al., 2015; Robson et al., 2016). Todas as organizações precisam de motivar e envolver os stakeholders, sejam formandos, pacientes, empregados ou consumidores.

Interessa saber como a gamification pode criar experiências envolventes, com o objetivo de resolver os problemas organizacionais. Num contexto de gestão, o engagement é uma questão-chave que recebe considerável atenção. Do ponto de vista psicológico, o engagement é uma experiência que compreende a energia, envolvimento e eficácia sentida pelo indivíduo durante a mesma (Maslach \& Leiter, 1997). O engagement dos trabalhadores refere-se ao seu envolvimento, satisfação e entusiasmo pelo trabalho (Kahn, 1990). O engagement pode estar positivamente associado com comprometimento organizacional e negativamente associado com desmotivação (Saks, 2006). Além disso, o aumento do envolvimento dos trabalhadores tem sido associado a um aumento da satisfação do cliente (Harter, Schmidt \& Hayes, 2002). No entanto, alcançar um elevado nível de engagement no trabalho é um desafio.

Aumentar a eficiência, a eficácia, a motivação e o engagement dos formandos em elearning pode ser alcançado por gamification. Atendendo à sua tecnologia, um dos campos onde a gamification pode ter maior impacto é na aprendizagem online (Domínguez et al., 2013). Daí, o uso da gamification no campo do e-learning estar a crescer e a ganhar popularidade.

\subsection{A gamification e a formação em hotelaria}

A hotelaria é uma indústria cujo sucesso e competitividade depende da qualidade das pessoas, não apenas dos gestores, mas de toda a equipa (Jones \& Haven, 2005). Portanto, é essencial que os hotéis desenvolvam práticas de GRH eficazes (Upamanyu, 2014).

As pessoas representam o capital mais valioso das organizações, visto que existe um potencial de vantagem competitiva nos conhecimentos, capacidades e atitudes dos trabalhadores que se refletem na qualidade do serviço prestado (Popescu \& Avram, 2012). Para tal, os gestores de RH devem focar-se sobre aspetos que atraiam bons profissionais, oferecendo um bom ambiente de trabalho, benefícios atraentes e progressão de carreira. Também é fundamental a utilização de ferramentas motivacionais de forma a obter altos níveis 
de desempenho. Outro dos aspetos essenciais é manter os trabalhadores mais talentosos através de políticas de retenção e de medidas que evitem a saída destes para empresas concorrentes.

Contudo, a hotelaria é muitas vezes caracterizada como um setor 'à parte' no que se refere às práticas de GRH. Keep e Mayhew (1999) resumem a lista de características da hotelaria: tendência para ordenados baixos; prevalência de horários de trabalho pouco ajustados ao equilíbrio entre a vida pessoal e a familiar; não implementação de políticas de igualdade de oportunidades; fraca ou inexistente progressão de carreira; práticas informais de recrutamento e contratação; não desenvolvimento de modelos formalizados de 'boas práticas' de GRH; inexistência de estudos de impacto da formação no desempenho dos trabalhadores da indústria hoteleira; inexistência de uma identidade sindical; elevados níveis de rotatividade laboral; dificuldades de recrutamento e de retenção.

Outros autores também caracterizam a indústria hoteleira como desprovida de uma estrutura de carreira clara, com alta rotatividade de pessoal e baixa formação (Hjalager \& Andersen, 2001; Maxwell, Watson \& Quail, 2004; Page, Brunt, Busby \& Connell, 2001). Muitas vezes os gestores veem o investimento em formação apenas como uma despesa, por terem receio que os trabalhadores deixem a empresa (Davies, Taylor \& Savery, 2001; Jameson, 2000; Loe, Ferrell \& Mansfield, 2000; Lowry, Simon \& Kimberley, 2002) e não a valorizam porque têm o seu tempo totalmente ocupado com o recrutamento e seleção. Contudo, os hotéis que não oferecem formação adequada apresentam maior rotatividade de pessoal (Lashley \& Best, 2002) e veem ameaçados os padrões de qualidade e o lucro.

Keep e Mayhew (1999) argumentam que a maior parte da formação que acontece no setor é impulsionada pela exigência legislativa e a maioria da formação desenvolvida é direcionada para que os trabalhadores se comportem de forma adequada com os clientes (Gilbert, Guerrier \& Guy, 1998).

Em termos históricos, as necessidades de formação em hotelaria foram vistas exclusivamente em termos de competências técnicas, e isto formou a base dos processos de educação e formação para o setor. O conteúdo do trabalho em hotelaria foi construído sob a base de uma acumulação de competências para determinadas tarefas técnicas. A educação e formação para o setor têm estado assentes na ligação à prática. As mudanças na natureza do trabalho, o impacto das tecnologias e as expetativas dos clientes forçaram uma reavaliação dos papéis, das competências técnicas e das competências transversais neste setor. Atualmente, a escassez de competências em hotelaria está mais ligada às competências transversais do que às competências técnicas específicas (Baum, 2002). No caso da hotelaria em Portugal, Calisto (2010) identifica a relevância que os empregadores atribuem às competências transversais.

A gamification pode contribuir para muitos dos problemas identificados nas práticas de $\mathrm{GRH}$ em hotelaria. A gamification pode orientar-se para o desempenho no recrutamento e seleção, garantindo um grupo qualificado de candidatos. Além disso, a gamification no processo formativo estimula o engagement na aprendizagem e o desenvolvimento dos trabalhadores, quer em termos de competências técnicas quer comportamentais. Estas dimensões de RH garantem o capital social necessário para fornecer serviços de alta qualidade. Mais ainda, segundo Negruşa et al. (2015), a experiência turística pode ser definitivamente influenciada, de forma indireta, através da introdução de gamification em atividades de RH. 


\section{Metodologia}

\subsection{Abordagem de investigação}

Este trabalho seguiu uma abordagem de investigação de natureza indutiva, assente numa estratégia de investigação essencialmente qualitativa e com um desenho de investigação exploratório, assente em dois sub-estudos: 1) estudo do lado da oferta de formação com recurso a gamification; e 2) estudo do lado da procura e das necessidades das empresas hoteleiras em termos de formação.

A metodologia de investigação utilizada, com recurso a entrevistas exploratórias e focus group, serviu o propósito de explorar o uso de gamification como uma ferramenta para a formação na hotelaria em Portugal. A decisão de realizar vários focus group em diferentes unidades hoteleiras possibilitou uma amostra diversificada, nomeadamente em termos dos diferentes níveis hierárquicos e das áreas habitualmente existentes em unidades hoteleiras.

\subsection{Objetivos}

Este estudo pretende explorar o uso de gamification como uma ferramenta para a formação na hotelaria em Portugal, quer do ponto de vista da oferta, identificando projectos formativos já desenvolvidos a nível europeu, e empresas sediadas em Portugal, que desenvolvam ferramentas tecnológicas, quer do lado da procura, caracterizando o potencial/necessidade da realidade hoteleira para o desenvolvimento destas ferramentas aplicadas à formação. Por outro lado, este projeto tem também como objetivo realizar o levantamento das principais necessidades formativas existentes nos estabelecimentos hoteleiros em Portugal e identificar os principais obstáculos percecionados pelos gestores e trabalhadores na execução do plano formativo dos estabelecimentos hoteleiros em Portugal. Finalmente, esta investigação procura também identificar as áreas/departamentos onde a aplicação da ferramenta seria mais adequada; e reconhecer o potencial da gamification aplicada à formação como uma estratégia motivacional e de adesão à formação no caso da hotelaria.

\subsection{Métodos e técnicas de recolha de dados}

Para a concretização do projeto foram utilizados um guião de entrevistas exploratórias e um guião para condução dos focus group.

Esta pesquisa é exploratória, uma vez que pretende analisar como os jogos podem ser usados na formação dos recursos humanos e como a unidade hoteleira pode beneficiar da gamification, como uma ferramenta ao desenvolvimento de soft skills, mas também de aquisição de competências técnicas. Como é considerada uma nova área e emergente, necessita de ser explorada em profundidade, e, portanto, seguiram-se paradigmas de pesquisa qualitativos. Os focus group têm sido frequentemente utilizados em investigação aplicada, no contexto das ciências sociais (Puchta \& Potter, 2004). Como método de pesquisa qualitativa, os focus group enfatizam a discussão, a partilha e a interacção, permitindo comparar experiências entre os participantes (Marshall \& Rossman, 2006).

As entrevistas exploratórias prendem-se com o objetivo de identificar empresas sediadas em Portugal que desenvolvam ferramentas tecnológicas com recurso a gamification para a área da hotelaria em Portugal. Este guião abrange os seguintes aspetos: a) projetos formativos com recurso a gamification; b) temas/conteúdos desenvolvidos nesse projetos; c) 
competências técnicas e/ou comportamentais a trabalhar; d) vantagens/desvantagens das ferramentas gamification; e) portefólio da empresa; f) temas/conteúdos desenvolvidos para a hotelaria; g) investimento/custo de um projeto formativo com recurso a estratégias gamification e; h) etapas de implementação de um projeto, incluindo duração e equipa do projeto.

O guião da entrevista para condução do focus group é de natureza semiestruturada, constituindo-se como uma preocupação central do investigador/moderador manter cada grupo na tarefa de obtenção das respostas para as questões previamente delineadas de acordo com os seguintes temas: 1 ) temas/áreas necessárias a integrar num plano formativo no grupo hoteleiro, na opinião de cada um dos intervenientes; 2) duração ideal para formação no contexto de aprendizagem online; 3) formato de aprendizagem ideal: em sala, online ou misto; 4) obstáculos que o setor hoteleiro coloca à aprendizagem e à formação; 5) satisfação em relação à formação realizada atualmente no sector hoteleiro; 6) motivação, refletindo o que motiva os trabalhadores para a formação e; 7) estratégias de melhoria para a eficácia da formação, para o aumento do grau de satisfação e da motivação para a formação.

No entanto, a construção do guião e a respetiva moderação privilegiou questões abertas, que incluíam as palavras ou temas-chave, centrando-se o papel do moderador no suporte ao grupo na exploração do tópico de tal forma que possam emergir novos insights face ao mesmo (Silva, Veloso \& Keating, 2014). É a estratégia do "funil" (Silva et al., 2014), em que à medida que a discussão avançou, as questões tornaram-se cada vez mais específicas ou, se quisermos, mais "afuniladas", sendo que as questões iniciais, ao serem mais genéricas, como referem Silva et al. (2014), ajudaram as pessoas a falarem e a pensarem sobre o tópico.

A moderação faz parte do processo, podendo a sua duração ir até as duas horas e meia (Stewart \& Shamdasani, 2007), embora, em média, se situe nos 90 minutos (Silva et al., 2014). A intervenção do moderador constitui um elemento chave nesta fase e, nesse sentido, as competências de moderação e de dinâmica de grupo que este possua representam elementos críticos do ponto de vista do seu sucesso.

Em cada focus group foi realizada uma contextualização do projeto, os seus objetivos, definição do conceito de gamification e a exemplificação com recurso a vídeo de um jogo na área do atendimento. Os participantes foram claramente informados sobre os objetivos do estudo e as regras de participação, incluindo tempo estimado de duração de modo a evitar abandonos precoces aquando da discussão em grupo (Silva et al., 2014).

Após esta apresentação, seguiu-se a exploração do guião iniciando com apresentação dos participantes e expetativas em relação ao tema. A discussão começou com um conjunto de questões pré-determinadas, com o objetivo central de obter respostas através da discussão ancorada nos temas previamente definidos no guião. A moderação teve como principal missão a condução e a manutenção da discussão. Teve assim como papel questionar, ouvir, manter a conversação no trilho e certificar-se que cada participante teve oportunidade de participar. Também teve como responsabilidade a gestão do equipamento de gravação e tomar notas sobre a discussão do grupo.

Estes focus group foram gravados com recurso a gravador e computador portátil, e posteriormente alvo de transcrição, sendo esta reprodução o mais fiel possível.

A complementaridade das transcrições com as notas que tinham sido recolhidas aquando da moderação dos focus group permitiu acrescentar qualidade às transcrições. Além disso, 
como sublinham Silva et al. (2014), as notas da moderadora são fonte de informações privilegiadas sobre expressões faciais, gestos, tom de voz e os contextos em que os discursos foram proferidos, fundamental no processo de descodificação, interpretação e análise de dados.

\subsection{Estudo do lado da oferta de formação gamified}

Para uma prospeção de projetos de gamification já desenvolvidos recorreu-se, em julho 2017, à plataforma Erasmus+ onde é possível aceder aos projetos europeus de aprendizagem ao longo da vida. Foram identificados os seguintes projetos formativos que se relacionam com o uso de ferramentas gamification na hotelaria ou no turismo em geral: 1) Touareg; 2) European catering \& hospitality training language course; 3) e-cuisine; 4) e-client e; 5) Green tourism.

Também se realizaram entrevistas exploratórias, entre janeiro e maio de 2017, com cinco empresas especializadas em ferramentas tecnológicas, aplicáveis à formação: 1) MagicBit; 2) Make a dream; 3) FractalMind; 4) Global Estratégias e; 5) Gfoundry.

\subsection{Estudo do lado da procura: grupos hoteleiros}

Do lado da procura analisou-se a dimensão do mercado (número de hotéis e número de profissionais), e identificaram-se projetos de gamification desenvolvidos em unidades hoteleiras e com aplicação à formação.

Para uma recolha de informação mais aprofundada sobre o lado da procura, foi utilizado, como explicado anteriormente, o método do focus group, de forma a aferir quais as funções e competências no âmbito da hotelaria em que a gamification seria mais pertinente.

$O$ estudo foi realizado com base nos casos de três cadeias hoteleiras portuguesas: 1) Grupo Pestana; 2) Grupo Nau; e 3) Grupo Hotéis Real. O Grupo Pestana é, atualmente, o maior grupo português no sector do turismo, sendo a sua cadeia hoteleira - Pestana Hotels \& Resorts, com 87 unidades e cerca de 10.000 quartos, a maior de origem portuguesa. $O$ Grupo NAU Hotels \& Resorts detém 10 hotéis de 4 e 5 estrelas, presente em três regiões distintas de Portugal (Lisboa, Alentejo e Algarve), três campos de golfe de 18 buracos e dois centros de congressos em Portugal. O Grupo Hotéis Real é um grupo hoteleiro português que conta com nove unidades nas regiões do Algarve e Lisboa.

\subsubsection{Focus group: caracterização da amostra}

Foram realizados cinco focus group. No primeiro estiveram presentes responsáveis da área de RH das três cadeias hoteleiras. O segundo grupo incluiu sete trabalhadores dos Hotéis Real, o terceiro abrangeu quatro trabalhadores do Grupo Nau, e o quarto incluiu cinco trabalhadores dos Hotéis Real. Foi constituído um quinto grupo com quatro estagiários do Grupo Pestana.

A amostra constituída foi fundamental para uma boa recolha de dados, pois representa diferentes unidades hoteleiras, trabalhadores com diferentes níveis de responsabilidade, diferentes papéis e de diferentes gerações, características que conduziram a discussões ativas e participadas por todos os elementos que integraram os grupos.

No que respeita ao género, a amostra é equilibrada, com onze participantes do género feminino e doze participantes do género masculino. Relativamente à faixa etária, temos uma cobertura abrangente das diferentes gerações, oito participantes entre os 16 e os 25 anos, oito participantes na faixa etária entre os 26 e 40 anos e sete participantes com mais de 40 anos. 
Os grupos constituídos, no que se refere aos níveis de responsabilidade, incluíram: três participantes da Direção, dez participantes pertencentes a um nível de chefia intermédia, seis com funções a um nível técnico/operacional e quatro estagiários.

\subsection{Tratamento e análise dos dados}

Após a realização das entrevistas exploratórias e focus group, procedeu-se à análise dos dados, utilizando o software NVivo. A análise decorreu ao longo de três subetapas: i) codificação/indexação: uma vez transcrito e (re)lido o texto, ocorreu um processo de atribuição de categorias (e, quando necessário, de subcategorias) refletindo estas os temas presentes no guião bem como os novos que emergiram da discussão dos grupos; ii) armazenamento/recuperação: esta fase é dedicada à compilação de todos os extratos do texto subordinados à mesma categoria de modo a poder compará-los, processo que foi realizado com recurso ao programa NVivo; iii) interpretação: suportada numa análise sistemática dos dados.

\section{Análise e discussão de resultados}

\subsection{Potencial da oferta - projetos e empresas de formação e gamification}

A partir da análise da plataforma Erasmus+, verifica-se que já tem sido explorado o potencial dos jogos para o mercado hoteleiro, numa vertente de formação técnica - idiomas (e-client, eurocathos), cozinha (e-cuisine) e atendimento. Também se identificaram projetos direcionados para a aquisição de competências comportamentais, como é o caso do TOUAREG, com o objetivo de treinar a comunicação, a socialização e também competências comportamentais de gestão. Por último, o projeto green tourism perspetiva mudanças de comportamento no domínio da ecologia.

A partir da identificação destes projectos, é visível que o conceito de jogo não é estranho ao setor do turismo. De acordo com a Organização Mundial do Turismo, os mecanismos de jogos têm a capacidade para criar experiências positivas no turismo: diversão, emoção, excitação, prazer, sensação de realização (Negruşa et al., 2015). No caso da hotelaria, as empresas podem combinar elementos de jogo e utilizá-los para induzir certos tipos de comportamento no cliente com consequências, por exemplo, na redução do consumo de energia e no desperdício de água, na utilização de transportes públicos ou na prática de avaliação dos serviços, etc. Além disso, as empresas podem usar os jogos para conduzir os seus trabalhadores a participar em processos de aprendizagem, melhorando a sua produtividade (Negruşa et al., 2015).

Muitas empresas já adotaram gamification para aumentar o envolvimento do cliente, ganhar a sua fidelidade, melhorar o desempenho dos empregados ou ganhar vantagens competitivas (Negruşa et al., 2015).

A título de exemplo, a gamification é aplicada na área da saúde, prevenção, terapia, educação e reabilitação, a fim de personalizar cuidados de saúde e tornar os processos nesse sector mais envolventes (McCallum, 2012). Healthmonth.com por exemplo, é um site que utiliza elementos do jogo, e uma combinação com a rede social para melhorar a saúde do jogador. 
Também na área de sustentabilidade existe um amplo número de exemplos de gamification para incentivar as pessoas a mudar seu comportamento no sentido de um estilo de vida mais sustentável (Volkswagen, 2009; Gamification Wiki, 2013 apud Xu et al., 2013).

Já no que se refere ao resultado das entrevistas realizadas com representantes das diferentes empresas com oferta gamification, denotam-se diferenças significativas no enfoque de cada uma delas, nomeadamente a nível da conceção. As propostas apresentadas por estas empresas permitiram ganhar conhecimento do mercado existente no que diz respeito à oferta de serviços formativos com recurso a estratégias de gamification. É um mercado com oferta diversificada no que respeita ao desenvolvimento do projeto, desde a tecnologia, à pedagogia, e ao design gráfico. Também foi possível identificar as fases de desenvolvimento de um projeto gamification e o tipo de perfis profissionais que integram as equipas de projeto nestas empresas. Outro ponto importante foi o conhecimento da dimensão temporal e do investimento necessário. $O$ desenvolvimento de um projeto de gamification tem a duração aproximada de três meses desde o planeamento à implementação. No que respeita ao investimento, o valor varia entre $3500 €$ e $9000 €$, dependendo do nível de complexidade do jogo, da ferramenta, dos cenários e dos conteúdos.

A partir das reuniões realizadas com as diferentes empresas, denotam-se diferenças significativas no enfoque de cada uma delas, nomeadamente a nível da conceção. Uma boa conceção deve fornecer informações que sejam: úteis, utilizáveis, desejáveis, passíveis de serem encontradas, acessíveis e credíveis (Usability.gov, 2014 apud Urh et al., 2015).

Em síntese, as reuniões realizadas, bem como as propostas apresentadas, permitiram ganhar conhecimento do mercado existente no que diz respeito à oferta de serviços formativos com recurso a estratégias de gamification. É um mercado com oferta diversificada no que respeita ao desenvolvimento do projeto, desde a tecnologia, à pedagogia, ao design gráfico. Também foi possível identificar as fases de desenvolvimento de um projeto gamification e o tipo de perfis dos profissionais que integram as equipas de projeto. Outro ponto importante foi o conhecimento da dimensão temporal e do investimento necessário. Por último, todas as propostas apresentaram a vantagem da criação de conteúdos modulares, e uma ferramenta tecnológica com a garantia de customização e à medida dos objetivos e resultados a alcançar. Os elementos importantes a considerar são a componente pedagógica, as opções de gamification, a componente tecnológica, o design, a modalidade de administração, bem como os elementos humanos e financeiros.

\subsection{Potencial da procura/necessidades de empresas hoteleiras - resultados dos focus group}

Na Figura 1 apresenta-se nuvem de palavras que salienta os termos mais utilizados. Para apresentação desta nuvem e a respectiva análise dos dados resultantes do focus group, foi utilizado o software NVivo, tendo resultado em sete categorias, associadas às questões definidas no guião de entrevista, e que foram colocadas nos focus group: 1) temas/áreas, 2) duração; 3) formato; 4) obstáculos; 5) satisfação; 6) motivação e; 7) estratégias. 
Figura 1. Nuvem de palavras

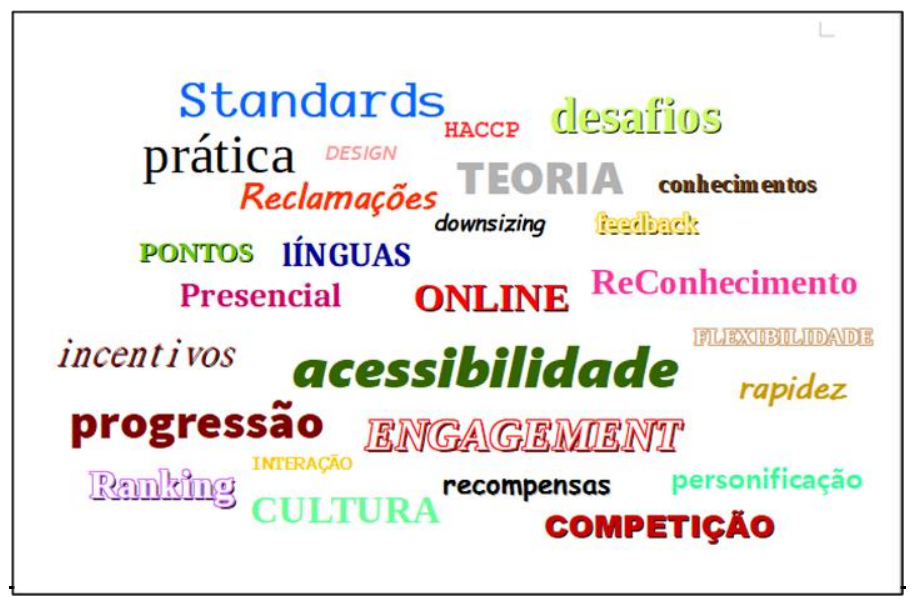

Os resultados dos focus group sugerem a adesão, por parte das unidades hoteleiras à metodologia deste projeto, o que é coerente com Robson et al. (2016) que refere que há um interesse crescente em gamification por parte das empresas, que estão continuamente a procurar novas maneiras que impactem na forma como se aprende e se influencia os comportamentos dos empregados e clientes.

Durante os focus group realizados, os conteúdos de aprendizagem identificados como mais necessários e ajustados à hotelaria incidiram sobre competências técnicas (idiomas, HACCP, sistema operativo, standards de serviço) mas também comportamentais (gestão de reclamações, liderança e gestão de equipas, comunicação). A duração mais adequada sugerida foi de, no mínimo, quinze minutos, no acesso rápido mobile, e de até quatro horas para a formação em sala. Para uma ferramenta desta natureza os participantes salientam a importância de um momento presencial de apresentação/ enquadramento da mesma e outro de encerramento como forma de balanço dos resultados obtidos.

A componente online deverá ser dedicada à aquisição de conteúdos teórico-práticos, com momentos de interação e competição entre os participantes, e baseada numa ferramenta acessível, dinâmica e atrativa no seu design. Erhel e Jamet (2013) também sugerem que a apresentação, tanto da informação na gamification, como das tarefas a serem realizadas, tem um grande impacto sobre a qualidade de processamento de informação dos formandos.

A categoria relativa a 'obstáculos' permite refletir sobre os desafios do projeto. A desmotivação, por questões relacionadas com as metodologias de aprendizagem: "muita teoria", "formação repetitiva", a obrigatoriedade da formação, mas também a realidade da operação hoteleira (falta de tempo e de recursos) - são aspetos aos quais a implementação deste projeto deve responder. Outros dos aspetos pertinentes são a falta de reconhecimento e a heterogeneidade das equipas (diferentes faixas etárias, diferentes bases de conhecimentos informáticos, diferentes habilitações e funções desempenhadas) que exigem que a ferramenta a desenvolver seja acessível a qualquer tipo de pessoa.

Esta realidade é coerente com a ideia de que um sistema gamified, permite a autonomia, isto é, a liberdade do jogador para aderir voluntariamente a um sistema gamified e abandonar a qualquer momento (Deterding, 2011; McGonigal, 2011; Schell, 2008 apud Xu et al., 2013). Os jogos normalmente permitem que os jogadores reiniciem ou joguem novamente, tornando os 
erros recuperáveis. Essa liberdade de falha permite aos formandos experimentar sem medo e aumenta o seu envolvimento (Lee \& Hammer, 2011 apud Urh et al., 2015).

Quando discutido o grau de satisfação e os fatores que o promovem, é reforçada por todos os grupos a importância da ligação da teoria à prática, com objetivos que devem ser muito precisos, bem como as regras, as orientações, os prazos, os requisitos e as limitações do e-learning. Efetivamente, a gamification facilita tornar os objetivos visíveis, o que motiva os formandos (Urh et al., 2015). É também fundamental a aplicabilidade dos conteúdos de aprendizagem, bom como o desenho de uma formação à medida. É importante a personificação de conteúdos e serviços com base numa previsão do que os utilizadores querem (Karpinskyj et al., 2014 apud Urh et al., 2015), o que aumenta a sua satisfação. Outros fatores de satisfação identificados referem-se à colaboração e interação entre os participantes, o que proporciona envolvimento e engagement.

A reflexão sobre os fatores de motivação incidiu fundamentalmente sobre a importância de a formação integrar as seguintes componentes: incentivos, recompensas, feedback, pontos, rankings e tabelas de classificação visíveis. Os desafios devem ser diários e proporcionar competição entre os participantes. Os prémios são críticos no jogo, pois proporcionam o retorno sob a forma de pontos alcançados, emblemas e níveis de competência do jogador. Estes laços envolvem cada vez mais o jogador, provocando diferentes tipos de emoções (esperança, medo, excitação) e conduz o jogador à ação, e torna-o motivado para o jogo (Zichermann \& Cunningham, 2011 apud Xu, et al., 2013). A existência de metas, regras, feedback e participação voluntária são elementos que, de acordo com McGonigal (2011), geram o sentimento de fluxo. $O$ feedback positivo é a base de gamification, aumenta a autoestima e a motivação dos utilizadores. O progresso e o status atual das atividades dos formandos devem ser apresentados de forma clara e graficamente. O sentimento de progresso dá aos formandos a motivação para o trabalho futuro. $O$ reconhecimento e a visibilidade são aspetos fundamentais no desenvolvimento de um projeto formativo, bem como a interação e comunicação entre os participantes.

A gamification cria um ambiente de aprendizagem que ativa motivações intrínsecas dos trabalhadores para aprender e progredir, eles podem satisfazer a sua curiosidade sobre diferentes temas, competir com outros colegas e adquirir autoestima e reconhecimento dos colegas pelas suas realizações. Por último, a reflexão dos grupos sobre as estratégias para se obter um grau de motivação elevado e, consequente adesão à formação, sugere que a ferramenta e os conteúdos de aprendizagem devem ser acessíveis a todos os elementos das equipas, atendendo às suas especificidades, características e competências. De facto, a compreensão dos desejos e motivações dos jogadores é a chave para projetar experiências gamified envolventes. Para maximizar o impacto de qualquer curso, os objetivos devem ser primeiramente especificados para atender às necessidades de aprendizagem (Landers \& Callan, 2011). Outro ponto assinalado é a flexibilidade e rapidez de acesso. Um jogo ao ser acessível a partir de qualquer lugar, pode permitir ao formando envolver-se com o material quando no local de trabalho não teria possibilidade; a relação entre a acessibilidade do jogo e os resultados da aprendizagem é mediado pela oportunidade (Landers \& Callan, 2011).

Nesta discussão também é abordada a vantagem desta ferramenta permitir o acesso a resultados, metadados, tracking pelos gestores como forma de analisarem a mudança de comportamentos, a aquisição de competências, e o grau de satisfação e motivação dos participantes. Sem métricas apropriadas, uma organização não pode medir o progresso ou 
melhorar experiências anteriores, ou seja, utiliza-se a gamification para acompanhar a pontuação (Robson et al., 2016). Também é assinalada a mais-valia deste projeto como uma forma, entre outras já existentes, de promover a cultura organizacional entre os participantes, de uma forma "natural e equilibrada". O aumento do envolvimento dos empregados tem sido associado a um aumento da satisfação do cliente (Harter et al., 2002), razão pela qual é do interesse dos gestores melhorar atributos comportamentais internos.

Todos os participantes referem as vantagens deste projeto associada às vantagens do elearning, aprendizagem em qualquer lugar e a qualquer hora, alcance geográfico, controle do formando (em termos de flexibilidade e conveniência) e custo efetivo num curso, coerente com Draves (2007).

\section{Conclusões}

O projeto concretizou os seguintes objetivos: a) identificou projetos formativos já desenvolvidos, a nível europeu, com recurso à gamification na área da hotelaria; bem como empresas sediadas em Portugal que desenvolvem ferramentas tecnológicas com recurso a gamification, ainda que não especificamente para a área da hotelaria; b) definiu as principais necessidades formativas existentes nas diferentes unidades, centradas em competências técnicas mas também comportamentais- standards de qualidade de serviço, idiomas, técnicas de vendas gestão de reclamações, entre outras; c) percecionou os principais obstáculos sentidos pelos gestores e trabalhadores na execução do plano formativo, como o downsizing das equipas, a rotatividade, a gestão de tempo e a desmotivação; e, d) identificou o potencial da gamification aplicada à formação como uma estratégia motivacional e de adesão à formação no caso da hotelaria.

A realização de entrevistas a diferentes empresas, com vertentes de desenvolvimento do negócio distintas, permitiu estabelecer comparações entre as diferentes propostas apresentadas. A decisão de realizar vários focus group em diferentes unidades hoteleiras possibilitou uma amostra comparativa, a diferentes níveis hierárquicos, e uma abrangência alargada das áreas existentes em unidades hoteleiras. Ainda que a dimensão da amostra (cinco focus group, três grupos hoteleiros, vinte e três participantes) possa considerar-se não representativa, a amostra constituída foi fundamental para uma boa recolha de dados, pois representam diferentes unidades hoteleiras, com diferentes níveis de responsabilidade, diferentes papéis e diferentes gerações, elementos que conduziram a discussões ativas e participadas por todos os elementos que a integraram.

A gamification possui elevado potencial para a melhoria da aprendizagem na educação e formação de profissionais. As organizações estão a adotar o e-learning através de um conjunto crescente de plataformas tecnológicas, das redes sociais, das tecnologias móveis e baseadas na web, que por sua vez mudam a forma como os indivíduos e as organizações participam, compartilham, cocriam, discutem e modificam qualquer tipo de experiência.

E pesquisas futuras poderão incluir: 1) a aplicação da gamification em diferentes setores do turismo; 2) aplicação da gamification para dentro (formação de trabalhadores) vs. para fora (o envolvimento do cliente); e 3) estudos empíricos sobre a eficácia da gamification para a experiência turística e a fidelização de clientes (Xu et al., 2014). De acordo com Gartner Inc (2013), cerca de $80 \%$ de todas as aplicações de gamification falham em satisfazer os seus objetivos devido à má conceção, pelo que será vantajoso existirem estudos que validem as 
diferenças nos resultados em função das características dos cursos gamificados. A implementação de sistemas de pontuação na vida real, pode levar a uma fadiga de coletar emblemas, pontos e troféus. Futuros estudos podem focar-se nos riscos do excesso de gamification na vida.

O conceito está a ser aplicado em vários setores, incluindo saúde, tecnologia da informação, e-commerce, entretenimento, educação e outros, com o objetivo de alcançar resultados organizacionais, como o envolvimento de empregados e clientes, aprendizagem e desenvolvimento, desempenho, recrutamento, fidelidade à marca, desenvolvimento da marca, participação do cliente e consciência social. No entanto, existe a necessidade de expandir e construir uma base teórica sólida para o conceito de modo a realizar todo o seu potencial na academia, bem como na indústria. Além disso, existe uma necessidade de abordar a falta de compreensão na construção de um design de jogo coerente e alinhado com resultados organizacionais para evitar falhas, tornando essencial a aplicação de um design de jogo para alcançar um resultado transformacional. Neste estudo, concluiu-se que a gamification em plataformas de e-learning possui potencial para aumentar a motivação dos formandos no contexto hoteleiro. No entanto, são necessários cuidados na conceção e implementação da experiência para que ela seja totalmente motivadora para os participantes. Este estudo é inovador, pois responde especificamente aos desafios que se colocam à hotelaria em Portugal, em termos da Gestão da Formação, identificando as vantagens de uma ferramenta de e-learning com recurso a estratégias de gamification (Hamari, Koivisto \& Sarsa, 2014).

Neste contexto, esta ferramenta pode constituir-se como um instrumento de potencialização da aprendizagem e do desenvolvimento das competências dos trabalhadores na área da hotelaria. A ferramenta a desenvolver pretende acompanhar as estratégias inovadoras do e-learning e aposta em novas abordagens pedagógicas que visam o desenvolvimento de competências profissionais com aplicação prática, rápida e eficaz. A interação com o conteúdo e a reflexão sobre a prática, ao serem uma constante, podem permitir ao formando apropriar-se dos conhecimentos e aplicá-los em situações que refletem a realidade. $\mathrm{O}$ formando reflete, decide e treina de modo construtivo. A linha pedagógica orientadora permitirá manter os trabalhadores ativos e envolvidos e atribuir ao formando o papel de ator na condução do seu processo formativo. As unidades hoteleiras poderão assim dispor de uma ferramenta de formação capaz de transmitir os conteúdos e missão junto do seu cliente interno, mantendo um canal de comunicação aberto de forma constante.

O estudo realizado é relevante quer do ponto de vista teórico, quer do ponto de vista prático. Em termos académicos, demonstra-se a aplicabilidade do conceito de gamification ao sector hoteleiro em Portugal, através de uma abordagem metodológica que pode ser replicada em outros contextos.

Do ponto de vista prático, o estudo fornece aos gestores de RH as vantagens da gamification como uma ferramenta para responder aos desafios que se colocam à hotelaria em Portugal, nomeadamente em termos de Gestão da Formação. Com base neste trabalho, poderá futuramente ser possível a construção e/ou adaptação de uma plataforma formativa/app-mobile com conteúdos específicos para a área Hoteleira com recurso a estratégias de gamification. 


\section{Referências}

Baum, T. (2002). Skills and training for the hospitality sector: A review of issues. Journal of Vocational Education \& Training, 54(3), 343-364. doi.10.1080/13636820200200204

Calisto, M. L. (2010, 23-24/09/2010). A importância das competências genéricas no recrutamento de diplomados do Ensino Superior: Estudo de casos em hotelaria e restauração. Comunicação apresentada em the Investigação e Intervenção em Recursos Humanos. Setúbal, Portugal.

Csikszentmihalyi, M. (1988). The flow experience and its significance for human psychology. In M. Csikszentmihalyi \& I. S. Csikszentmihalyi (Eds.), Optimal experience: Psychological studies of flow in consciousness (pp. 15-35). Cambridge: Cambridge University Press.

Csikszentmihalyi, M. (2008). Flow: The psychology of optimal experience. Nova lorque: HarperCollins.

Davies, D., Taylor, R. \& Savery, L. (2001). The role of appraisal, remuneration and training in improving staff relations in the Western Australian accommodation industry: A comparative study. Journal of European Industrial Training, 25(7), 366-373. doi:10.1108/EUMo000000005837

Deterding, S., Sicart, M., Nacke, L., O’Hara, K. \& Dixon, D. (2011). Gamification. using game-design elements in non-gaming contexts. In Proceeding of $\mathrm{CHI}$ '11 extended abstracts on human factors in computing systems (pp. 2425-2428). Vancouver, Canadá.

Domínguez, A., Saenz-de-Navarrete, J., de-Marcos, L., Fernández-Sanz, L., Pagés, C. \& Martínez-Herrálz, J.J. (2013). Gamifying learning experiences: Practical implications and outcomes. Computers \& Education, 63, 380-392. doi:10.1016/j.compedu.2012.12.020

Draves, W. A. (2007). Advanced teaching online. Wisconsin: Learning Resources Network (LERN).

Elverdam, C. \& Aarseth, E. (2007). Game classification and game design: Construction through critical analysis. Games and Culture, 2(1), 3-22. doi:10.1177/1555412006286892

Enz, C. A. \& Siguaw, J. A. (2000). Best practices in human resources. Cornell Hotel and Restaurant Administration Quarterly, 41(1), 48-61.

Erhel, S. \& Jamet, E. (2013). Digital game-based learning: Impact of instructions and feedback on motivation and learning effectiveness. Computers \& Education, 67, 156-167. doi:10.1016/j.compedu.2013.02.019

Gartner Inc. (2013). Gartner says by 2014, 80 percent of current gamified applications will fail to meet business objectives primarily due to poor design, 2012. Disponivel em http://www.gartner.com/newsroom/id/2251015

Gilbert, D., Guerrier, Y. \& Guy, J. (1998). Sexual harassment issues in the hospitality industry. International Journal of Contemporary Hospitality Management, 10(2), 48-53. doi:10.1108/09596119810207183

Hamari, J., Koivisto, J. \& Sarsa, H. (2014). Does gamification work? A literature review of empirical studies on gamification. In 47th Hawaii International Conference System Sciences (pp. 3025-3034).

Harter, J. K., Schmidt, F. L. \& Hayes, T. L. (2002). Business-unit-level relationship between employee satisfaction, employee engagement, and business outcomes: A meta-analysis. Journal of Applied Psychology, 87(2), 268-279. doi:10.1037//0021-9010.87.2.268

Hays, R. T. (2005). The effectiveness of instructional games: A literature review and discussion. (Technical report 2005-004). Orlando: Naval Air Warfare Center Training Systems Division.

Hjalager, A.-M. \& Andersen, S. (2001). Tourism employment: Contingent work or professional career? Employee Relations, 23(2), 115-129. doi:10.1108/01425450110384165

Jackson, S. A. \& Eklund, R. C. (2002). Assessing flow in physical activity: The flow state scale-2 and dispositional flow scale-2. Journal of Sport \& Exercise Psychology, 24(2), 133-150. doi:10.1123/jsep.24.2.133

Jameson, S. M. (2000). Recruitment and training in small firms. Journal of European Industrial Training, 24(1), 43-49. doi:10.1108/03090590010308255

Jones, E. \& Haven, C. (2005). Tourism SMEs, service quality, and destination competitiveness. Wallingford: CABI.

Kahn, W. A. (1990). Psychological conditions of personal engagement and disengagement at work. Academy of Management Journal, 33(4), 692-724.

Keep, E. \& Mayhew, K. (1999). The leisure sector skills task force research. (Paper No. 6, DFEE). Londres: Publications.

Kelly, T. (2012). Real gamification mechanics require simplicity and, yes, game designers can do it. Disponível em https://techcrunch.com/2012/12/08/real-vs-fake-gamificationmechanics/?guccounter=1 
Kietzmann J. H., Hermkens K., McCarthy I. P. \& Silvestre, B. S. (2011) Social media? Get serious! Understanding the functional building blocks of social media. Business Horizons, 54(3), 241-251. doi: 10.1016/j.bushor.2011.01.005

Kim, W. C. \& Mauborgne, R. A. (2014). Blue ocean leadership. Harvard Business Review, 92(5), 60-72.

Landers, R. N. (2014). Developing a theory of gamified learning: Linking serious games and gamification of learning. Simulation \& Gaming, 45(6), 752-768. doi:10.1177/1046878114563660

Landers, R. N. \& Callan, R. C. (2011). Casual social games as serious games: The psychology of gamification in undergraduate education and employee training. In M. Ma, A. Oikonomou \& L. Jain (Eds.), Serious games and edutainment applications (pp. 399-423). Londres: Springer.

Lashley, C. \& Best, W. (2002). Employee induction in licensed retail organisations. International Journal of Contemporary Hospitality Management, 14(1), 6-13. doi:10.1108/09596110210415060

Lazzaro, N. (2004). Why we play games: Four keys to more emotion without story. In Proceedings of the Game Developers Conference (pp.1-8). São Francisco, EUA.

LeBlanc, M. (2004). Game design and tuning workshop. Paper presented at FuturePlay 2005 International Academic Conference on the Future of Game Design and Technology, East Lansing, Michigan.

Liu, M., Horton, L., Olmanson, J. \& Toprac, P. (2011). A study of learning and motivation in a new media enriched environment for middle school science. Educational Technology Research and Development, 59(2), 249-265. doi 10.1007/s11423-011-9192-7

Loe, T. W., Ferrell, L. \& Mansfield, P. (2000). A review of empirical studies assessing ethical decision making in business. Journal of Business Ethics, 25(3), 185-204. doi:10.1023/A:1006083612239

Lowry, D. S., Simon, A. \& Kimberley, N. (2002). Toward improved employment relations practices of casual employees in the New South Wales registered clubs industry. Human Resource Development Quarterly, 13(1), 53-70. doi:10.1002/hrdq.1013

Marshall, C. \& Rossman, G. B. (2006). Designing qualitative research (4. Ed.). Thousand Oaks: Sage.

Maslach, C. \& Leiter, M. P. (1997). The truth about burnout: How organizations cause personal stress and what to do about it. São Francisco: Jossey-Bass.

Maxwell, G., Watson, S. \& Quail, S. (2004). Quality service in the international hotel sector: A catalyst for strategic human resource development? Journal of European Industrial Training, 28(2/3/4), 159182. doi:10.1108/03090590410527591

McCallum, S. (2012). Gamification and serious games for personalized health. Stud Health Technol Inform, 177(2012), 85-96.

McGonigal, J. (2011). Reality is broken: Why games make us better and how they can change the world. Nova lorque: Penguin.

Negrușa, A. L., Toader, V., Sofică, A., Tutunea, M. F. \& Rus, R. V. (2015). Exploring gamification techniques and applications for sustainable tourism. Sustainability, 7(8), 11160-11189. doi:10.3390/su70811160

Page, S., Brunt, P., Busby, G. \& Connell, J. (2001). Tourism: A modern synthesis. Londres: Thomson Learning. Popescu, C. \& Avram, D.-M. (2012). New trends in human resource management in the hospitality industry. In the International Conference of Scientific aper AFASES 2012 (pp. 185-190), Brasov, Roménia.

Puchta, C. \& Potter, J. (2004). Focus group practice. Londres: Sage.

Randstad Work Solutions (2007). The world of work 2007.

Robson, K., Plangger, K., Kietzmann, J. H., McCarthy, I. \& Pitt, L. (2015). Is it all a game? Understanding the principles of gamification. Business Horizons, 58(4), 411-420. doi:10.1016/j.bushor.2015.03.006

Robson, K., Plangger, K., Kietzmann, J. H., McCarthy, I. \& Pitt, L. (2016). Game on: Engaging customers and employees through gamification. Business Horizons, 59(1), 29-36. doi:10.1016/j.bushor.2015.08.002

Saks, A. M. (2006). Antecedents and consequences of employee engagement. Journal of Managerial Psychology, 21(7), 600-619. doi: 10.1108/02683940610690169

Silva, I. S., Veloso, A. L. \& Keating, J. B. (2014). Focus group: Considerações teóricas e metodológicas. Revista Lusófona de Educação, (26), 175-190.

Simmons, K. (2008). Intergenerational communication in the workplace. The Online Journal for Certified Managers. May/ June 2008.

Stewart, D. W. \& Shamdasani, P. N. (2007). Focus groups: Theory and practice. Thousand Oaks: Sage publications.

Turismo de Portugal. (2017). Turismo em números. Disponível em https://travelbi.turismodeportugal.pt/pt-pt/Paginas/turismo-em-numeros-2017.aspx 
Upamanyu, S. (2014). Managing Attrition through HR System in Hotel Industry. International Journal of Multidisciplinary Approach and Studies, 1(6), 510-522.

Urh, M., Vukovic, G., Jereb, E. \& Pintar, R. (2015). The model for introduction of gamification into e-learning in higher education. Procedia-Social and Behavioral Sciences, 197, 388-397. doi:10.1016/j.sbspro.2015.07.154

Volkswagen (2009). The fun theory.

Xu, F., Weber, J. \& Buhalis, D. (2014, January 21-24). The gamification of tourism. In Z. Xiang \& I. Tussyadiah (Eds.), Information and Communication Technologies in Tourism 2014: Proceedings of the International Conference in Dublin (pp. 525-537). Wien: Springer.

CARLA PATRÍCIA VIEIRA é licenciada em Psicologia, mestre em Psychological Empowerment e em Gestão Hoteleira e pós-graduada em Direção e Gestão de Recursos Humanos. Em 2018, desenvolveu o Projeto Gamification e Formação Hoteleira na ESHTE. Iniciou a sua experiência profissional, em 2007, como formadora nas áreas da Comunicação, Empregabilidade e Liderança e Gestão de Equipas. Em 2008, assumiu funções de coordenação e gestão formativa no IEFP, e desde 2012 até à atualidade, assume a responsabilidade de gestão da formação dos psicólogos e da gestão das pessoas, na Ordem dos Psicólogos Portugueses. Endereço institucional: Escola Superior de Hotelaria e Turismo do Estoril, Avenida Condes de Barcelona, 808, 2769-510 Estoril, Portugal.

MARIA DE LURDES CALISTO é doutorada em Gestão, especialização em Recursos Humanos, pela Universidade de Évora e mestre em Políticas de Desenvolvimento dos Recursos Humanos pelo ISCTE IUL. É Professora Adjunta na Escola Superior de Hotelaria e Turismo do Estoril, onde também coordena e colabora em diversos projetos de investigação. É membro integrado do CiTUR - Centro de Investigação, Desenvolvimento e Inovação em Turismo e colaboradora do CEFAGE - Centro de Estudos e Formação Avançada em Gestão e Economia. Os seus interesses de investigação centram-se nas áreas da estratégia, capital humano, inovação e intraempreendedorismo, no contexto das empresas de serviços, nomeadamente de Hotelaria e Turismo. Possui larga experiência no contexto empresarial, na área da Formação e da Gestão de Recursos Humanos. Endereço institucional: Escola Superior de Hotelaria e Turismo do Estoril, Avenida Condes de Barcelona, 808, 2769-510 Estoril, Portugal.

NUNO GUSTAVO é Professor-Adjunto na Escola Superior de Hotelaria e Turismo do Estoril. É doutorado em Turismo, Lazer e Cultura e mestre em Lazer e Desenvolvimento Local pela Universidade de Coimbra. Tem como área de ensino e investigação a gestão hoteleira, a gestão de operações e distribuição turística, a gestão de evento e o turismo de saúde, lecionando ao nível dos vários ciclos do ensino superior. Tem participado em diversos projetos de formação, consultoria, investigação e planeamento no domínio da Hotelaria e Turismo em Portugal, Moçambique e Cabo Verde. Do seu curriculum destaca-se ainda a colaboração com instituições de ensino internacionais e nacionais, como a Grenoble Graduate School of Business e a Universidade Católica do Porto-EGE. Endereço institucional: Escola Superior de Hotelaria e Turismo do Estoril, Avenida Condes de Barcelona, 808, 2769-510 Estoril, Portugal.

Submetido em 1 novembro 2018

Aceite em 14 março 2019 